\title{
Drug-Coated Balloon and In-Stent Restenosis: A Clinical Strategy to Manage Coronary Artery Diseases
}

\author{
Prima Hapsari Wulandari ${ }^{1 *}$ \\ ${ }^{1}$ Affiliation - Faculty of Medicine, Airlangga University, Indonesia \\ *Corresponding email: Prima Hapsari Wulandari.Email: primahapsari@outlook.com
}

\begin{abstract}
Cardiovascular events are the most common cause of morbidity and mortality worldwide. Coronary artery diseases, including myocardial infarctions, are prevalent among all populations. There are multiple management strategies for coronary artery diseases, but stent implantation is among the most effective interventions. Nevertheless, some patients develop restenosis of the intervened vessel, and the number constitutes about 10\%-20\% of all patients. In-stent restenosis leads to adverse effects that might end in sudden cardiac death. Restenosis forges a clinical challenge for practicing physicians. A drug-coated balloon has propitious potential and is showing results comparable to stents implantation to overcome in-stent restenosis. This review aims to evaluate the effectiveness and advancement of drug-coated balloons in treating in-stent restenosis as a coronary intervention, along with its benefits and drawbacks.
\end{abstract}

Keywords - stent implantation, in-stent restenosis, coronary artery disease, drug-coated balloon.

\section{INTRODUCTION}

Coronary artery diseases (CADs) are the first cause of death worldwide. The condition results from partially or completed occlusion of one or more coronary arteries. Subsequently, the area supplied by the occluded vessel dies, leading to muscle necrosis, which disrupts the heart's electrical activity and pumping capacity. As a complication, an arrhythmia occurs and imposes a direct cause of death [1-2].

Multiple treatment strategies and management plans for CADs have been developed, including the administration of thrombolytic therapy, especially when the patient presents in the golden first four hours. The facility's capacity involved a role in the management; a center for interventional radiology guaranteed the application of percutaneous coronary intervention (PCI). PCI intervention includes a wide range of management strategies. It includes percutaneous 
transluminal coronary angioplasty (PTCA), stent implantation, and drug-coated balloons (DCBs) [3]. There is a rapid improvement in treatment strategies, even at the level of bioengineering technologies. In practice, intervention choice depends on the patient's condition, facility capacity, possible procedure complications, and several other factors that influence practitioners' preference for stent implantation over PTCA. The restenosis rate in PTCA may reach up to 50\%. On the other hand, the restenosis rate in drug-coated stent implantation is about 10-20\% [4-5]. There are many reported adverse events with in-stent restenosis (ISR), and the presence of these complications is called major adverse cardiovascular events (MACEs). It comprises a group of symptoms that resemble angina pectoris, acute myocardial infarction, and sudden cardiac death.

The use of stents to manage CADs started after 1970. The restenosis complication might also occur with the balloon dilation technique. Balloon dilation works as elastic recoil of the vessel's internal lamina layer. Studies investigating the use of balloon technique in managing CADs reported a rate of restenosis of 30-60\% when patients were followed up [6]. The ISR occurred three and six months after the procedure. In the 1980s, bare-metal stents (BMSs) were first used and started their development. BMS procedure was an evolution as it decreased the restenosis rate to less than $30 \%$ [6-7]. Although there are several efforts to reduce the restenosis complication, it still occurs. Practitioners sometimes use certain antiproliferative drugs such as paclitaxel and sirolimus to reduce ISR incidence [8]. The insertion of these drugs around the stent is called drugeluting stents (DESs).

It was reported that ISR is less prevalent in DESs use when compared to BMSs. However, the implantation of stents and the management of CADs remain substantial clinical challenges [9]. The choice of treatment technique never fails to raise controversy among practitioners because the options are dependent on the availability of equipment and the patients' characteristics. Many studies that have discussed the use of drug-coated balloon (DCB) angioplasty and compared it to DES concluded that both are very similar in the complication of ISR [10-12]. Thus, it is imperative to be cautious when selecting management to reduce complications in the patients.

\section{REVIEW}




\section{Method}

Multiple methods have been used to maintain a high-quality review; databases such as Google Scholar and PubMed were thoroughly searched. Keywords like stent implantation, in-stent restenosis, coronary artery disease, drug-coated balloon were applied. Related studies to the main object and review articles were included. Included persons consisted of men and women between the ages of 18 and 80, excluding adolescents children. All studies incorporated are published in English. This investigation procedure revealed 33 appraised articles.

\section{DISCUSSION}

PCI intervention includes a wide range of management strategies. It comprises percutaneous transluminal coronary angioplasty (PTCA), stent implantation, and drug-coated balloons (DCBs) [3]. There is a rapid improvement in treatment strategies, to the point of bioengineering technologies. The intervention choice depends on the patient's condition, facility capacity, and possible procedure complications in clinical practice. Most physicians prefer stent implantation over PTCA because the restenosis rate in PTCA may reach up to 50\%. Even though the rate of restenosis in drug-coated stent implantation is about 10-20\% [4-5], many reported adverse events occur with in-stent restenosis (ISR), especially three and six months after the procedure. When bare-metal stents (BMSs) were developed to reduce the restenosis complication, ISR still manifests as the main complication. Physicians sometimes prescribe antiproliferative drugs such as paclitaxel and sirolimus to reduce the incidence of ISR [8]. The insertion of these drugs around the stent is called drug-eluting stents (DESs). Many studies that have discussed the use of drugcoated balloon (DCB) angioplasty and compared it to DES concluded that both are very similar in the complication of ISR [10-12].

\section{In-Stent restenosis}

There are several definitions of ISR, but the agreed one is the occlusion of at least $50 \%$ of the vessel lumen caused by a new proliferative lesion on top of a stent or involving $5 \mathrm{~mm}$ adjacent to the stent seen the coronary angiography [9-10]. It is reported that ISR occurs after a successful PCI procedure. The complication materializes on average one year after the procedure following failures to maintain the vessel's patency, which resulted from either restenosis or new thrombosis. The difference is that the restenosis process is gradual, and the thrombus is acute. Therefore, choosing the procedure is critical, and the patient should be the center of care in the choice. Some 
studies mentioned that the restenosis might occur three months following the procedure [11]. The patient presents with symptoms of myocardial infarction, chest pain, and angina pectoris. The condition is actual for about $10 \%$ of patients [12-13]. The goal of the management is to restore the vascularization.

Physicians classified the ISR into two categories according to the pattern of occlusion. Type one is the restenosis within less than $10 \mathrm{~mm}$ length to the side of the stent, which is called focal or localized lesions. Type one is further classified into sub-types called focal multiple or diffuse, which is a lesion of more than $10 \mathrm{~mm}$ of length or not confined to the stent edges. Type two of the lesion is confined to the stent edges. Lastly, there is a diffuse type that extends over the stent edges, which is type three. Type four completely occludes the stent.

\section{ISR Pathology}

It is known that ISR pathology results from the proliferation of smooth muscle cells and the infiltration of macrophages at the site of the stent. A high amount of elastic fibers are found in the wall of arteries, especially the coronary arteries; hence it is essential to provide recoil characteristics to the vessels as they distend [14]. When an artery is occluded as the PCI procedure is performed, the recoil occurs within seconds. When the vessel wall undergoes hyperplasia, restenosis occurs. The vessel wall hyperplasia arises from various causes, such as an injury that leads to inflammatory response and subsequent lumen narrowing at the stent site. The pathophysiology of the new restenosis is the same as vessel thrombus and platelets play the most significant role in this pathology [15].

The management of ISR is conducted by inserting a balloon at the stent site and inflating it to contrast stenosis. The procedure will dilate the vessel lumen again [15-16]. However, in some cases where tears existed in the vessel's intimal and media layers, there should be an increase in the balloon's inflation to decrease the minimal luminal diameter (MLD) of the stenosis [16]. The process of restenosis takes months to develop, and in most cases, it is completed in 6 months. The redistribution of proliferative cells throughout the vascular wall causes some patients to present at an earlier or later period from the first procedure. The process is dependent on the rapidity of the cells' redistribution [16].

Table 1. Time-related assessment of in-stent restenosis (ISR) 


\section{Early (within days)}

Elastic recoil

Relocation of axially transmitted plaque

\section{Late (weeks to months)}

Reorganization of thrombus

Neointima proliferation

Cell proliferation

Cell migration

Cell matrix synthesis

Remodeling

Resolution of inflammation

\section{New Atherosclerosis}

When restenosis occurs, it is attributed to new atherosclerotic changes in the vessel wall. Restenosis is defined as the new accumulation of lipid-laden macrophages foam cells within the stent in the occluded vessel. The complication occurs with or without the formation of necrotic tissue or new calcification in the vessel wall [17]. Restenosis is mostly initiated by a mechanical injury to the arterial wall, which stretches it and makes the vessel more susceptible to atherosclerosis. The presence of previous risk factors of increasing LDL-cholesterol increases the rate of new atherosclerosis. While it is known that atherosclerosis takes years to develop for the first time, atherosclerosis can rapidly advance at the stent site. The phenomenon is due to the fact that endothelium is already damaged by the original injury that required the first stent implantation. Additionally, the damage may be induced by the original PCI procedure. In other cases where DESs is used, the antiproliferative drugs might play a role in making the condition worse [18]. Hence, there is a growing need to use intravascular imaging and optical coherence tomography. The implementation of intravascular imaging and optical coherence tomography is potential to deliver a higher rate of accurate diagnosis of the underlying problem in stent failure [18].

\section{Clinical Management}

The clinical management of ISR developed and changed over the years since the introduction of stents in 1970. There are two recent meta-analyses focused on which strategy is the best management modality to treat ISR. The first one was conducted by Siontis et al. [19], which included 27 trials and 5923 patients followed up at six months and one year. The authors reported that DES was found to be the most superior technique statistically. The primary outcome of the study was to measure the percent of restenosis and the secondary outcome is to look at the 
complications. DCB was in second place in managing the ISR. The second meta-analysis conducted by Giacoppo et al. [20] included 24 trials and 4880 patients. The primary outcome is the lumen loss determined by angiography. The results were similar to the first meta-analysis in which DCB and DES were the superior techniques to other modalities. However, late lumen loss was found to be lower when using DCB compared to DES.

\section{Evidence-Based Practice Of Using DCBs}

The introduction of DCB to the management of CADs and ISR makes it easier to administer antiproliferative agents without leaving any additional stent strut layers. It has been reported that this modality is useful in patients with BMS-ISR and DES-ISR. Table 2 shows the clinical trials conducted to investigate the drug-coated balloon technique. The studies revealed that DCB is superior to plain old balloon angioplasty (POBA). When patients were followed at six months, it was found that angiographic results were better in patients who underwent DCB. Another study examined and compared the effectiveness of paclitaxel-DES and paclitaxel-coated DCB. The authors found that DCB reduced the late angiographic loss more significantly than the other technique. The RIBS V trial concluded that late angiographic findings in patients undergoing DES were lower than patients who underwent DCB. Accordingly, we can conclude that most studies and trials recommended the use of DCB to be superior to all other techniques in managing CADs and ISR 21-24].

On the other hand, several studies assessed the use of DCB in patients with DES-ISR. The studies demonstrated that DCB is superior in both clinical and angiographic results when compared to POBA alone. The result was approved by another trial in which patients had DES-ISR and underwent DCB. Furthermore, another study concluded that DCB is almost equivalent to paclitaxel-DES in patients with DES-ISR. The patients in the study had a lumen loss of $0.46 \mathrm{~mm}$ at one year follow up [25-27].

Eventually, we can say that DCB proves reliable effectiveness when compared to most other modalities. Table 2 shows more trials and their results. A recent study that assessed ISR of DES patients compared the second-generation DES versus DCB treatment. The second-generation DES is Paclitaxel- and Everolimus-Eluting Stent. The study found that results are favoring the use of second-generation DES over DCB. However, the controversy follows on the use of which modality. It is also still unknown whether the DCB efficacy can be improved by lesion preparation 
using a scoring system. The questions mentioned above will be discussed in the ongoing ISARDESIRE 4 randomized trial.

Table 2. A comparison table of studies involving the clinical trials conducted to investigate the drug-coated balloon technique with their subsequent findings.

\begin{tabular}{|c|c|c|}
\hline Reference & Trial name & Findings \\
\hline 21 & PACCOCATH ISR I & $\begin{array}{l}\text { At 6-month follow-up, LLL: } 0.03 \pm 0.48 \mathrm{~mm} \text { versus } \\
0.74 \pm 0.86 \mathrm{~mm}(\mathrm{P}=0.002) \text {; TLR } 23 \% \text { versus } 0 \%\end{array}$ \\
\hline $22-23$ & PACCOCATH ISR II & $\begin{array}{l}\text { At } 6 \text { or } 12 \text { months, LLL: } 0.81 \pm 0.79 \mathrm{~mm} \text { versus } 0.11 \\
\pm 0.46 \mathrm{~mm}(\mathrm{P}<0.001) ; 37 \% \text { versus } 4 \%\end{array}$ \\
\hline 24 & PEPCAD II & $\begin{array}{l}\text { At } 6 \text { months, LLL: } 0.17 \pm 0.42 \mathrm{~mm} \text { versus } 0.38 \pm 0.61 \\
\mathrm{~mm}(\mathrm{P}=0.03) ; 6.3 \% \text { versus } 15.4 \%\end{array}$ \\
\hline 25 & ISAR-DESIRE3 & $\begin{array}{l}\text { At } 9 \text { months, diameter of in-stent restenosis: } 38 \% \text { : } \\
37.4 \% \text { versus } 54.1 \% \text { (noninferiority } \mathrm{P}=0.007 \text { ) }\end{array}$ \\
\hline 26 & PEPCAD-DES & $\begin{array}{l}\text { At } 6 \text { months, LLL: } 0.43 \pm 0.61 \mathrm{~mm} \text { versus } 1.03 \pm 0.77 \\
\mathrm{~mm}(\mathrm{P}<0.001)\end{array}$ \\
\hline 27 & PEPCAD China ISR & $\begin{array}{l}\text { At } 9 \text { months, LLL: } 0.46 \pm 0.51 \mathrm{~mm} \text { versus } 0.55 \pm 0.61 \\
\mathrm{~mm} \text { (noninferiority } \mathrm{P}<0.0005 \text { ) }\end{array}$ \\
\hline 28 & DELUX registry & At 6 and 12 months, MACE: $8.5 \%$ and $15.1 \%$ \\
\hline 29 & Habara et al. & $\begin{array}{l}\text { At } 6 \text { months, LLL: } 0.18 \pm 0.45 \mathrm{~mm} \text { versus } 0.72 \pm 0.55 \\
\mathrm{~mm}(\mathrm{P}=0.001)\end{array}$ \\
\hline 30 & $\begin{array}{l}\text { SeQuent Please World } \\
\text { Wide Registry }\end{array}$ & At 9 months, TLR: $9.6 \%$ versus $3.8 \%(\mathrm{P}<0.001)$ \\
\hline 31 & Valentines I & At 8 months, MACE: $0: 23.8 \%(\mathrm{P}=0.002)$ \\
\hline 32 & PEPPER & $\begin{array}{l}\text { At } 6 \text { months, LLL: } 0.05 \pm 0.28 \mathrm{~mm} \text { versus } 0.19 \pm 0.29 \\
\mathrm{~mm}(\mathrm{P}=0.001)\end{array}$ \\
\hline 33 & RIBS IV & $\begin{array}{l}\text { At 3-years follow-up, MLD: } 2.03 \pm 0.7 \mathrm{~mm} \text { versus } 1.8 \\
\pm 0.6 \mathrm{~mm}\end{array}$ \\
\hline
\end{tabular}

Note: LLL, late lumen loss; TLR, target lesion revascularization; DES, drug-eluting stent; MACE, major adverse cardiovascular events; MLD, minimal lumen diameter. 


\section{CONCLUSION}

The introduction of new methods to manage CADs has significantly reduced the incidence rate of ISR. However, the management of ISR is still challenging for physicians. The current systematic review suggests that DES and DCB are the most superior techniques in managing CADs with the least ISR rates. When a patient needs more than two metal stents in the same ISR lesion, the need increases the long-term detrimental outcomes, even with newer DCB that proves superior to manage ISR. There is a need for further research and trials to spotlight the role of these current management techniques and modalities to improve patients' clinical outcomes and provide a guide for practicing physicians.

\section{DISCLOSURE}

\section{Funding}

None.

\section{Conflict of Interest}

The author declares there is no conflict of interest regarding all aspect of the study.

\section{Author Contribution}

PHW is responsible for the study from the literature search, data gathering, data analysis, until reporting the results of the study by a narrative form of literature review.

\section{REFERENCES}

1. S. Garg, C. Bourantas, and P. W. Serruys, "New concepts in the design of drug-eluting coronary stents," Nature Reviews Cardiology, vol. 10, no. 5, pp. 248-260, 2013.

2. P. Scacciatella, M. D'Amico, M. Pennone et al., "Effects of EPC capture stent and CD34+ mobilization in acute myocardial infarction," Minerva Cardioangiol, vol. 61, no. 2, pp. 211219, 2013.

3. M. Nobuyoshi, T. Kimura, H. Nosaka et al., "Restenosis after successful percutaneous transluminal coronary angioplasty: serial angiographic follow-up of 229 patients," Journal of the American College of Cardiology, vol. 12, no. 3, pp. 616-623, 1988.

4. S. Mohan and A. Dhall, "A comparative study of restenosis rates in bare metal and drugeluting stents," International Journal of Angiology, vol. 19, no. 2, pp. e66-e72, 2010. 
5. S. J. Park, S. J. Kang, R. Virmani, M. Nakano, and Y. Ueda, "In-stent neoatherosclerosis," Journal of the American College of Cardiology, vol. 59, no. 23, pp. 2051-2057, 2012.

6. M. S. Lee, A. Pessegueiro, R. Zimmer, D. Jurewitz, and J. Tobis, "Clinical presentation of patients with in-stent restenosis in the drug-eluting stent era," The Journal of Invasive Cardiology, vol. 20, no. 8, pp. 401-403, 2008.

7. B. Schnorr, U. Speck, and B. Scheller, "Review of clinical data with Paccocath- coated balloon catheters," Minerva Cardioangiologica, vol. 59, no. 5, pp. 431-445, 2011.

8. M. Taniwaki, G. G. Stefanini, S. Silber et al., "4-Year clinical outcomes and predictors of repeat revascularization in patients treated with new-generation drug-eluting stents," Journal of the American College of Cardiology, vol. 63, no. 16, pp. 1617-1625, 2014.

9. G. D. Dangas, B. E. Claessen, A. Caixeta, E. A. Sanidas, G. S. Mintz, and R. Mehran, "Instent restenosis in the drug eluting stent era," Journal of the American College of Cardiology, vol. 56, no. 23, pp. 1897-1907, 2010.

10. R. Mehran, G. Dangas, A. S. Abizaid et al., "Angiographic patterns of in-stent restenosis," Circulation, vol. 100, no. 18, pp. 1872-1878, 1999.

11. J. Zhu and J. Ge, "GW26-e1581 a meta-analysis of randomized controlled trials of plain old balloon angioplasty versus drug eluting balloon in patients with in-stent restenosis," Journal of the American College of Cardiology, vol. 66, no. 16, p. C232, 2015.

12. F. Alfonso, M. J. P' erez-Vizcayno, B. Garc'1a del Blanco et al., "Comparison of the efficacy of everolimus-eluting stents versus drug-eluting balloons in patients with in-stent restenosis (from the RIBS IV and V randomized clinical trials)," the American Journal of Cardiology, vol. 117, no. 4, pp. 546-554, 2016.

13. M. S. Lee and G. Banka, "In-stent restenosis," Interventional Cardiology Clinics, vol. 5, no. 2, pp. 211-220, 2016.

14. M. C. Serrano, A. K. Vavra, M. Jen et al., "Poly (diol-co-citrate)s as novel elastomeric perivascular wraps for the reduction of neointimal hyperplasia," Macromolecular Bioscience, vol. 11, no. 5, pp. 700-709, 2011.

15. H. D. Danenberg, F. G. P. Welt, M. Walker, P. Seifert, G. S. Toegel, and E. R. Edelman, "Systemic inflammation induced by lipopolysaccharide increases neointimal formation after balloon and stent injury in rabbits," Circulation, vol. 105, no. 24, pp. 2917-2922, 2002. 
16. P. K. Shah, "Inflammation, neointimal hyperplasia, and restenosis," Circulation, vol. 107, no. 17, pp. 2175-2177, 2003.

17. F. Otsuka, R. A. Byrne, K. Yahagi et al., "Neoatherosclerosis: overview of histopathologic findings and implications for intravascular imaging assessment," European Heart Journal, vol. 36, no. 32, pp. 2147-2159, 2015.

18. G. Nakazawa, F. Otsuka, M. Nakano et al., "The pathology of neoatherosclerosis in human coronary implants bare-metal and drug-eluting stents," Journal of the American College of Cardiology, vol. 57, no. 11, pp. 1314-1322, 2011.

19. GC. Siontis, GG. Stefanini, D. Mavridis et al. "Percutaneous coronary interventional strategies for treatment of in-stent restenosis: a network metaanalysis," Lancet. 2015;386:655-664.

20. D. Giacoppo, G. Gargiulo, P. Aruta, P. Capranzano, C. Tamburino, D. Capodanno, "Treatment strategies for coronary in-stent restenosis: systematic review and hierarchical Bayesian network meta-analysis of 24 randomised trials and 4880 patients," British Medical Journal. 2015;351:h5392.

21. B. Scheller, C. Hehrlein, W. Bocksch et al., "Treatment of coronary in-stent restenosis with a paclitaxel-coated balloon catheter," New England Journal of Medicine, vol. 355, no. 20, pp. 2113-2124, 2006.

22. B. Scheller, C. Hehrlein, W. Bocksch et al., "Two year followup after treatment of coronary in-stent restenosis with a paclitaxel-coated balloon catheter," Clinical Research in Cardiology, vol. 97, no. 10, pp. 773-781, 2008.

23. B. Scheller, Y. P. Clever, B. Kelsch et al., "Long-term follow-up after treatment of coronary in-stent restenosis with a paclitaxel-coated balloon catheter," JACC: Cardiovascular Interventions, vol. 5, no. 3, pp. 323-330, 2012.

24. M. Unverdorben, C. Vallbracht, B. Cremers et al., "Paclitaxelcoated balloon catheter versus paclitaxel-coated stent for the treatment of coronary in-stent restenosis," Circulation, vol. 119, no. 23, pp. 2986-2994, 2009.

25. R. A. Byrne, F. J. Neumann, J. Mehilli et al., "Paclitaxel-eluting balloons, paclitaxel-eluting stents, and balloon angioplasty in patients with restenosis after implantation of a drug-eluting stent (ISAR-DESIRE 3): a randomised, open-label trial," The Lancet, vol. 381, no. 9865, pp. 461-467, 2013. 
26. H. Rittger, J. Brachmann, A. M. Sinha et al., "A randomized, multicenter, single-blinded trial comparing paclitaxel-coated balloon angioplasty with plain balloon angioplasty in drugeluting stent restenosis," Journal of the American College of Cardiology, vol. 59, no. 15, pp. 1377-1382, 2012.

27. B. Xu, R. Gao, J. A. Wang et al., “A prospective, multicenter, randomized trial of paclitaxel coated balloon versus paclitaxel-eluting stent for the treatment of drug-eluting stent in-stent restenosis," JACC: Cardiovascular Interventions, vol. 7, no. 2, pp. 204-211, 2014.

28. R. Toelg, B. Merkely, A. Erglis et al., "Coronary artery treatment with paclitaxel-coated balloon using a BTHC excipient: clinical results of the international real-world DELUX registry," Euro Intervention, vol. 10, no. 5, pp. 591-599, 2014.

29. S. Habara, K. Mitsudo, K. Kadota et al., "Effectiveness of paclitaxel-eluting balloon catheter in patients with sirolimuseluting stent restenosis," JACC: Cardiovascular Interventions, vol. 4, no. 2, pp. 149-154, 2011.

30. J. W“ ohrle, M. Zadura, S. M“ obius Winkler et al., "SeQuent please world wide Registry, prospective registry study," Journal of the American College of Cardiology, vol. 60, no. 18, pp. 1733-1738, 2012.

31. P. Stella, A. Belkacemi, R. Waksman et al., "the valentines trial: results of the first one week worldwide multicentre enrolment trial, evaluating the real world usage of the second generation DIOR paclitaxel drug-eluting balloon for in-stent restenosis treatment," Euro Intervention, vol. 7, no. 6, pp. 705-710, 2011.

32. C. Hehrlein, U. Dietz, J. Kubica et al., "Twelve-month results of a paclitaxel releasing balloon in patients presenting with instent restenosis first-in-man (PEPPER) trial," Cardiovascular Revascularization Medicine, vol. 13, no. 5, pp. 260-264, 2012.

33. F. Alfonso, M. J. P' erez-Vizcayno, J. Cuesta et al., "RIBS IV study investigators (under the auspices of the interventional Cardiology working group of the Spanish society of Cardiology). 3-Year clinical follow-up of the RIBS IV clinical trial: a prospective randomized study of drug-eluting balloons versus everolimus-eluting stents in patients with in-stent restenosis in coronary arteries previously treated with drug-eluting stents," JACC: Cardiovascular Interventions, vol. 11, no. 10, pp. 981-991, 2018. 